\title{
Occupational Stress and its Relation to Insomnia among Nurses of Educational Hospitals in Qazvin
}

\author{
Zohreh Yazdi ${ }^{1}$, Alireza Haji seid Javadi², Mehrzad Afsari ${ }^{3}$ Mahnaz Abbasi ${ }^{*}$ \\ ${ }^{1} \mathrm{MD}$, associate professor, Social Determinants of Health Research Center, Qazvin University of Medical Sciences \\ ${ }^{2} \mathrm{MD}$, associate professor, Metabolic Disease Research Center, Qazvin University of Medical Sciences \\ ${ }^{3} \mathrm{MD}$, Qazvin University of Medical Sciences \\ ${ }^{4} \mathrm{MD}$, associate professor, Metabolic Disease Research Center, Qazvin University of Medical Sciences
}

\begin{abstract}
Stress is an inevitable part of life. It is a universal phenomenon and general experience of human which is necessary for his/ her survival and growth. It affects everyone irrespective of age, gender, race, economical condition or educational level. Although stress can be natural and necessary, if it is strict, continuous and repetitious and an individual is not able to respond to it effectively or if supportive sources are limited, it becomes a negative phenomenon which brings about physical and psychological disorders. The present study aimed to determine occupational stress level and conditions causing stress among nurses of educational hospitals in Qazvin Province. This is a sectional study on 194 nurses working in health care centers of Qazvin Province. Demographic questionnaire and Philip Rice's occupational stress questionnaire were completed by the nurses. In addition, they were requested to answer four more questions about insomnia and then the data was collected and analyzed using SPSS statistical software. Occupational stress has very high prevalence among nurses. The study showed that $91 / 2 \%$ of them experienced high levels of occupational stress. There is a significant relation between wards and occupational stress. In addition, the most important stressors among the nurses were recognized and the ways they respond to them were also studied. Moreover, the relation between the nurses' occupational stress and insomnia was examined. This study showed that the level of occupational stress among nurses was significant and there was a clear and meaningful relation between stress and the ward they work in. Among the stressors were physical tiredness due to work, emergency situations, heavy workloads and performing tasks requiring less knowledge and last but not least having multiple chiefs. It was also found out that nurses' occupational stress played a pivotal role in their insomnia status.
\end{abstract}

Keywords: Occupational stress, nurses, insomnia status, confronting with stress

\section{INTRODUCTION}

Stress is an inevitable and necessary part of life which is a universal phenomenon and general experience of human necessary for his/her survival and growth (Wienger,

*Correspondence: dr.mabbasiayahoo.com

Mahnaz Abbasi, Qazvin University of medical sciences, Qazvin, Iran

Fax number: 00982833359503 Tel number: 0982833365386

Received: 10 March 2018 Accepted: 01 June 2018

Sleep and Hypnosis

Journal homepage:

www.sleepandhypnosis.org

ISSN: 2458-9101 (Online)
Hange, Bjorkelund, \& Ahlborg, 2015; Krakow, et al., 2007). It affects everyone irrespective of age, gender, race, economical condition or educational level. While some stresses are natural and necessary, if it is strict, continuous and repetitious or if an individual is not able to give a proper response to it or if supportive sources are limited, it is considered a negative phenomenon which can cause physical and psychological disorders (Dahal, Kumar, \& Thapa, 2015; Cheung, \& Yip, 2015).

Work as an integral part of human life can be a major source of stress. Occupational stress is a health hazard that stems from a number of factors in modern workplace Not only it threatens occupational health, but also it is a 
disincentive factor from economical point of view (Moustaka, \& Constantinidis, 2010; Sarafis, Rousaki, Tsounis, \& Malliarou, et al., 2016).

Unfavorable physical conditions, health risks, workload, time-limit pressure, responsibilities, ambiguity and conflict in roles, conflict with colleagues and senior managers and subordinates, constraints, low participation in decision making, job promotion or degradation, and lack of job security are amongst common job-related stressors (Li, Cheng, Zhu, 2017; de Paiva, Canario, de Paiva China, \& Goncalves, 2017). According to World Health Organization more than $50 \%$ of workers in industrialized countries complain about workplace stress (Moustaka, \& Constantinidis, 2010; Kortum, Leka, \& Cox, 2010). Only in USA about 11 million people suffer from occupational stress. Another survey showed that nearly a quarter of Americans consider occupational stress as the largest and most important problem in life (GarciaVelazques, Jokela, \& Rosenstorm, 2017; Pabayo, Fuller, Goldstein, Kawachi, Gilman, 2017).

Nursing by its very nature is associated with high levels of stress. It requires high level skills, teamwork in different situations, providing 24-hour care and the resulting emotional burdens can bring about a wide range of occupational stressors (Amanya, Nakitende, \& Ngabirano, 2016; Kwiatosz, Fijałkowska-Nestorowicz, Fijałkowska, Aftyka, \& Kowalczyk, 2017).

Nursing is composed of a series of activities and interpersonal relations that are often stressful. Having to accept responsibilities that one is not prepared for, working with people with no qualification, conflicts with colleagues, working in environments where supervisors and managers do not support employees are some issues which contribute to work-related stress (Borges, Moreira, \& Andrade, 2017; Portela, et al. 2015). The stress; obviously, increases by the expectations from nurses. For example, nurses who are experiencing negative emotions and reactions in their personal life are expected to use positive and supportive behavior and verbal expression in their roles at work (Borges, Moreira, \& Andrade, 2017; Kuma, 2013).

A study conducted in Taiwan found high levels of occupational stress, anxiety, and insomnia among nurses working in rotational shift work (Shen, Yen, Yang, \& Lee,
2013). A cohort study done in Brazil in some hospitals of Rio de Janeiro aimed to investigate the level and results of occupational stress on nurses, found that $45 / 8 \%$ of the whole participants had insomnia complaints. Statistically there was a significant relation between occupational stress and stressful life events with insomnia complaints (Robaina, et al. 2009).

Studies have shown that $7 / 4 \%$ of nurses make absence because of fatigue or disability due to stress which is $80 \%$ higher than other occupational groups (Borges, Moreira, \& Andrade, 2017; Portela, et al. 2015; Shen, Yen, Yang, \& Lee, 2013). Some other studies have found that job satisfaction has a strong relation with stress reduction in turn plays a pivotal role in nurses' quality of work life (Amanya, Nakitende, \& Ngabirano, 2016; Portela, et al. 2015; Shen, Yen, Yang, \& Lee, 2013; Khamisa, Oldenburg, Peltzer, \& llic, 2015).

No study has been conducted in Iran on examining different situations which can cause stress to nurses and their relation to insomnia.

According to the above considerations and the importance of this issue, the present study aimed to investigate frequency of occupational stress and the factors producing it among nurses in Qazvin University of Medical Sciences.

\section{METHOD}

The present descriptive study is analytical-descriptive epidemiological study to evaluate the prevalence and factors of occupational stress among nurses in Qazvin training hospitals. In this study all shift work nurses in Qazvin health care hospitals who were willing to cooperate were studied. This cross-sectional study was done in teaching hospitals of Qazvin university of Medical Sciences. The nurses having at least one year work experience were studied in this research.

All the nurses participated in this study were asked to complete questionnaires for collecting information. This questionnaire contains demographic information about the age, sex, education, height and weight and the work place. Following this questionnaire questions were asked about nurses' insomnia status. 
Philip L. Rice questionnaire approved by the America Mental Health Institute with the reliability of 0.92 , was used in this study. The questionnaire included 57 questions and three subscales: interpersonal relationships, physical health and occupational interests. Initial questions of this questionnaire have been developed to assess problems in interpersonal relationships and job satisfaction or dissatisfaction. Then the physical conditions which cause individual's daily fatigue have been dealt with and the third part of the questionnaire was dedicated to the occupational interests. A 5-point scoring scale testing is done in this test. Using the answer key is performed for final scoring and the score of occupational stress obtained from the total scores. The results of quantifying all the questions are added together which is identifier of individuals' occupational stress score. Occupational stress score less than 116 indicates lower stress levels, 140-116 indicates moderate stress and more than 140 indicates high levels of stress (Rice, 1992; Roostaee, Nikmanesh, Sharifi-Rad, Kiani, Shahnazi, 2016).

This scale has been translated in Farsi and validated by Hatami (1998).

The insomnia severity questionnaire has 5 questions which estimate the severity of insomnia during last 2 weeks. Its score ranges from 0 to 28 . The higher score demonstrates the higher risk of insomnia and need for medical assistance. The questionnaire was used by Morine and his coworkers for the first time (Morin, 1993). The Kronbach' alpha of the questionnaire in Farsi was 0.76 in the previous study which is acceptable. (Yazdi,
Sadeghniiat, Zohal, Elmizadeh, 2012)

After announcing readiness and collecting consent letters the questionnaires were given to them to be completed. All data were entered into SPSS software version 16 and were analyzed.

\section{RESULTS}

The study population comprised 194 nurses working in teaching hospitals of Qazvin University of Medical Sciences with the average age of $6.3 \pm 31.5$ (minimum 22 and maximum 49 years). There were 14 male nurses (7.2\%) and the rest were female. The mean body mass index was $3.8 \pm 24.6$.

Of all participants, 34,52, 48, 20 and 40 nurses worked in the sectors of internal medicine, surgery, emergency, pediatrics and intensive care unit, respectively.

Table 2 shows the number of nurses in each group of low, medium and high occupational stress in various domains, and in the questionnaires.

Total scores of participants were in the range of 255116 and the mean scores of nurses participating in the study was 177.4 with a standard deviation of 2.84 .

Since only one person was in the range of low stress, the participants were divided in two groups of high stress and medium stress in the range of 140 . Therefore, 17 people i.e. $8.8 \%$ were reported with moderate stress and 177 people i.e. $91.2 \%$ were reported with high stress levels.

Table 1. Nurses' answers in each of the subscales of occupational stress questionnaire

\begin{tabular}{lcccc}
\hline & Interpersonal relationship & Physical conditions & Occupational interest & Total \\
\hline Average & 76.94 & 71.90 & 29.36 & 117.41 \\
Standard deviation & 12.64 & 14.82 & 6.43 & 28.45 \\
Minimum & 49 & 35 & 11 & 116 \\
Maximum & 117 & 108 & 45 & 255 \\
\hline
\end{tabular}

Table 2. Number of nurses in each of the groups of low, medium and high occupational stress

\begin{tabular}{lccc}
\hline & Low stress & Normal stress & High stress \\
\hline Interpersonal relationship & $2(1 \%)$ & $22(11.3 \%)$ & $170(87.6 \%)$ \\
Physical condition & $10(5.1 \%)$ & $19(9.8 \%)$ & $165(85 \%)$ \\
occupational interest & $5(2.5 \%)$ & $26(13.4 \%)$ & $163(84 \%)$ \\
total & $1(0.5 \%)$ & $16(8.2 \%)$ & $177(91.2 \%)$ \\
\hline
\end{tabular}


Table 3. Relationship between stress with demographic variables and nurses sleep

\begin{tabular}{|c|c|c|c|}
\hline & Low stress & High stress & P-value \\
\hline Age & 30.41 & 31.61 & 0.459 \\
\hline BMI & 25.49 & 24.49 & 0.298 \\
\hline \multicolumn{4}{|l|}{ Gender } \\
\hline male & 1 & 13 & 0.82 \\
\hline female & 16 & 164 & \\
\hline \multicolumn{4}{|l|}{ Wards } \\
\hline internal & 9 & 45 & 0.016 \\
\hline surgery & 8 & 132 & \\
\hline \multicolumn{4}{|c|}{ Difficulty in initiation of sleep } \\
\hline yes & 4 & 84 & 0.06 \\
\hline no & 13 & 93 & \\
\hline \multicolumn{4}{|c|}{ Difficulty in continuing of sleep } \\
\hline yes & 8 & 96 & 0.51 \\
\hline no & 9 & 81 & \\
\hline \multicolumn{4}{|c|}{ Difficulty in early morning awakening } \\
\hline yes & 5 & 87 & 0.11 \\
\hline no & 12 & 90 & \\
\hline \multicolumn{4}{|c|}{ Sleep quality } \\
\hline good & 7 & 125 & 0.013 \\
\hline poor & 10 & 52 & \\
\hline
\end{tabular}

Table 4. Relationship between occupational stress and insomnia status of nurses with level of occupational stress

\begin{tabular}{lcccc}
\hline & \multicolumn{3}{c}{ p-value } \\
\cline { 2 - 5 } & Interpersonal relationship & Physical condition & Occupational interest & Total \\
\hline Beginning of sleep & 0.373 & 0.186 & 0.429 & 0.238 \\
During sleep & 0.765 & 0.452 & 0.048 & 0.347 \\
Awakening & 0.246 & 0.013 & 0.229 & 0.027 \\
Sleep quality & 0.025 & 0.003 & 0.001 & 0.001 \\
\hline
\end{tabular}

Table 3 shows the link between stress with demographic variables and nurses' sleep.

The relationship between occupational stress and insomnia status of nurses with different levels of occupational stress and the analysis of results using Pearson correlation can be seen in the table 4 .

As observed, high stress has a significant relationship with poor quality of sleep (0.001). Likewise, stress in domain of physical condition is associated with waking up earlier in the morning (0.013) and occupational stress in the job interests are associated with repeated waking during sleep (0.048).

\section{DISCUSSION}

Occupational stress is a challenge in all jobs and nursing is snot an exception. In addition to contribution to different health issues, it can cause serious problems for health care systems in general. The results of a study by Abdi et al. showed that more attention needs to be paid to symptoms of occupational stress among nurses which include depression, separation from patients, absence from work, and work quality reduction (Abdi, \& Shahbazi, 2001). Social protection of nurses and helping them in stress management at workplace needs be on the agenda to reduce stress and its damaging effects. In order to retain the quality of health care and to prevent the adverse effects of occupational stress, sources of stress must be identified and necessary actions must be taken to eliminate or reduce them (Beheshti, \& Hajizadeh, 2015; Kayalha, Yazdi, Rastak, \& Dizaniha, 2013).

The findings of this study showed nurses' occupational stress in terms of age, gender, level of education, work place ward as $91.2 \%$ with high stress levels and the $8.8 \%$ with medium or low levels of stress which according to the criteria of high, medium and low is at high level. Since 
nursing is a stressful job in nature, this result was not unexpected. In a study by Rezaee et al. while nurses indicated stress as a major cause of leaving job, it was not among the five causes of leaving job known by employers (Rezaii, Hosseini, \& Fallahi, 2006; Gheshlagh, et al., 2017). If employees are satisfied with their occupational stress balance and the balance between their work environment and life, they will have a tendency not to leave their job and even recommend others to work for their employers. In their study $59 \%$ of case studies were found to have high levels of occupational stress (Rezaii, Hosseini, \& Fallahi, 2006; Gheshlagh, et al., 2017).

In another study on nurses in Zanjan, 57.4\%, 40\% and $2.6 \%$ of nurses were reported to have high, medium and low levels of stress respectively (MortaghyGhasemy, Ghahremani, Azimi, \& Ghorbani, 2010).

According to findings of another study done by Khodaveisi et al. on occupational stress among nurses in Hamadan hospitals $51 / 3 \%$ were reported to have stress while others had no stress (Khodaveisi, Mohammadi, \& Omidi, 2006). In a similar study by Rahimi et al., stress levels and the effective factors on nurses' occupational stress in some hospitals of Tehran were investigated where $44 / 1 \%$ of them had high stress, $54 / 1 \%$ and $1 / 8 \%$ had moderate and low levels of stress respectively (Rahimi, Ahmadi, \& Akhond, 2004). Findings of a research done by Rezaee et al., aiming the relation between occupational stress and nurses social support showed that $59 \%$ of nurses had high levels of stress and $37 \%$ of them had moderate stress levels (Rezaee, Behbahany, Yarandy, \& Hosseine, 2006).

In our study using Philip Rice questionnaire it was shown that interpersonal relations, numerous chiefs, lack of determination power on future of their work, performing tasks that requires less knowledge and education, and fatigue due to high workload and emergency situations are among the most important stressors in nursing. Likewise, high level of tiredness when leaving workplace which was habitual among $40 / 7 \%$ of the nurses reduced job interest and increased stress level in such a way that $57 / 7 \%$ of nurses were not sure about choosing the same occupation again if they had another chance.
Lu and his colleague in examining occupational stress and the related factors among nurses found that heavy workload and responsibility were major sources of nurses' occupational stress (Lu, Chang, \& Wu, 2007).

Similarly, the findings of the present study showed that nurses' occupational stress correlates with their insomnia status of nurses so that with an increase in stress level, the insomnia status becomes significantly worse and the individual tends to wake up earlier in the morning than required which in turn contributes to morning anxiety (Abdi, \& Shahbazi, 2001; Kayalha, Yazdi, Rastak, \& Dizaniha, 2013).

The results of this study showed that nurses working in Qazvin training hospitals experience high levels of stress and that among the most common contributing factors are working in emergency departments, heavy workload, workrelated fatigue and having to work with multiple bosses.

An increase in the number of nursing personnel and holding specialized training courses for them on time management and stress management is recommended to help reduce their vocational stress (Lu, Chang, \& Wu, 2007; Rezaee, Behbahany, Yarandy, \& Hosseine, 2006).

Since the sampling of this study is non-probabilistic, the results cannot be generalized to the whole community of nurses but the present study and similar studies can act as a guide to reduce occupational stress among nurses and consequently cause improvement in sleep status of these hard-working and painstaking individuals. It is recommended that the study will be done in larger populations consisting of private and non-private hospitals and the results will be compared.

Acknowledgment: Hereby I acknowledge valuable guidance of my venerable professor Dr. Zohre Yazdi. I also sincerely thank all the nurses participating in this study and also all those who helped us in this way.

Conflict of interest: The authors declare no conflict of interest.

Informed consent: Informed consent was obtained from all the participants included in the study.

Funding: The authors declare that the current study was not financially supported by any institution or organization. 
Yazdi / Sleep and Hypnosis 2019

\section{References}

Abdi, H., Shahbazi, L. (2001). Occupational stress in nursing working in ICU and its relation to burnout. Journal of Shahid Sadughi University of Medical Sciences, 9(3), 64-70.

Amanya, S. B., Nakitende, J., Ngabirano, T. D. (2017). A crosssectional study of stress and its sources among health professional students at Makerere University, Uganda. Nursing Open. 4,5(1), 70-76.

Beheshti, M. H., Hajizadeh, R. (2013). Workload, occupational stress, and general health of female employees of public welfare centers. Journal of Occupational Health and Epidemiology, 2(4), 174-182.

Borges, J., Moreira, T., \& Andrade, D. (2017). Nursing care interpersonal relationship questionnaire: elaboration and validation. Revista Latino-Americana de Enfermagem. 25,e2962, 1-10.

Cheung, T., \& Yip, P. (2015). Depression, anxiety and symptoms of stress among Hong Kong nurses: a cross-sectional study. International Journal Environment Research Public Health, 12.

Dahal, H., Kumar, S., \& Thapa, T. (2018). Prevalence and risk factors of post-traumatic stress disorders among the survivors of 2015 Nepal Earthquake, in Dhading, Nepal. Sleep and Hypnosis, 20(2): 128-139.

De Paiva, L. C., Canario, A. C. G., de Paiva China, E. L. C., Goncalves, A. K. (2017). Burnout syndrome in health-care professional in a university hospital. Clinics (SaoPaulo), 72(5), 305-309.

Garcia-Velazquez, R., Jokela, M., Rosenstorm, T. H. (2017). Symptom severity and disability in psychiatric disorders: The U.S. Collaborative Psychiatric Epidemiology Survey. Journal Affective Disorders, 222, 204-210.

Gheshlagh, R., Parizad, N., Dalvand, S., Zarei, M., Farajzadeh, M., Karami, M., \& Sayehmiri, K. (2017). The prevalence of job stress among nurses in Iran: a meta analysis study. Nursing and Midwifery Studies, 6, 143-148.

Hatami, M. (1998). Determination of stress on working mothers and non-working mothers and effective of therapist reduce of stress, [Dissertation]. Tehran: Allame Tabatabai University.

Kayalha, H., Yazdi, Z., Rastak, S., \& Dizaniha, M. (2013). Obvious and hidden anxiety and the related factors in operating room nurses employed in general hospital, Qazvin, Iran: a crosssectional study. Global Journal of Health Science, 5(6), 202208

Khamisa, N., Oldenburg, B., Peltzer, K., Dragan, I. (2015). Work related stress, burnout, job satisfaction and general health of nurses. International Journal of Environmental Research and Public Health, 12, 652-666.

Khodaveisi, M., Mohammadi, N., Omidi, A. (2006). Frequency of job stress in clinical nurses. Scientific Journal of Hamadan Nursing \& Midwifery Faculty, 13(2): 44-54.

Kortum, E., Leka, S., \& Cox, T. (2010). Psychological risks and work-related stress in developing countries: health impact, priorities, barriers and solutions. International Journal of Occupational Medicine and Environmental Health, 23(3), 225238.

Krawkow, B., Haynes, P., Warner, T., Melendrez, D., Sisley, B., Johnston, L., ...,\& Lee, S. (2007). Clinical sleep disorder profiles in a large sample of trauma survivors: an interdisciplinary view of posttraumatic sleep disturbance. Sleep and Hypnosis, 9(1): 6-15.

Kumar, R. (2013). Psychosomatic complaints among nursing students-A cross sectional survey from Punjab. Delhi Psychiatry Journal, 16(2), 343-349.
Kwiatosz-Muc, M., Fijatkowska-Nestorowicz, A., Fijałkowska, M., Aftyka, A., \& Kowalczyk, M. (2017). Stress prevalence and stressors among anaesthesiology and intensive care unit workers: A multicentre survey study. Australian critical care, Article in Press.

Li, H., Cheng, B., \& Zhu, X. P. (2017) .Quantification of burnout in emergency nurses: A systematic review and meta-analysis. International Emergency Nursing. Article in press.

Morin, C. M. (1993). Insomnia: psychological assessment and management. New York, NY: Guilford Press.

Lu, K., Chang, L., Wu, H. (2007). Relationship between professional commitment, job satisfaction, and work stress in public health nurses in Taiwan. Journal of Professional Nursing, 23, 110-116.

MortaghyGhasemy, M., Ghahremani, Z., Azimi, A., \& Ghorbani, F. (2010). Nurses job stress in therapeutic educational centers in Zanjan. Journal of Research Development in Nursing \& Midwifery, 8(1): 42-51.

Moustaka, E., \& Constantinidis, T. (2010). Sources and effects of work-related stress in nursing. Health Science Journal, 4(4), 210-216

Pabayo, R., Fuller, D., Goldstein, R. B., Kawachi, I., Gilman, S. E. (2017). Income inequality among American states and the conditional risk of post-traumatic stress disorder. Social Psychiatry and Psychiatric Epidemiology, 52(9), 1195-1204.

Portela, L., Luna, C., Rotenberg, L., Costa, A., Toivanen, S., Araujo, T., \& Griep, R. (2015). Job strain and self-reported insomnia symptoms among nurses: what about the influence of emotional demands and social support? BioMed Research International. http://dx.doi.org/10.1155/2015/820610.

Rahimi, A., Ahmadi, F., \& Akhond, M. (2004). An investigation of amount and factors affecting nurses' job stress in some hospitals in Tehran. Hayat, 10 (3), 13-22.

Rezaii, S., Hosseini, A., \& Fallahi, M. (2006). Evaluating impact of communication skills training on level of occupational stress among nursing personnel working at rehabilitation centers in cities: Ray- Tehran- Shemiranat. Tehran University of Medical Journal, 64(1), 21-26.

Rezaee, N., Behbahany, N., Yarandy, A., \& Hosseine, F. (2006). Correlation between occupational stress and social support among nurses. Iran Journal of Nursing, 19(46), 71-78.

Rice, P. L. (1995). Stress and health. Canada: Thomson Publication. Robaina, J., Lopes, C., Rotenberg, L., Faerstein, E., Fischer, F., Moreno, C., ..., Chor, D. (2009) Stressful life events and insomnia complaints among nursing assistants from a university hospital in Rio de Janeiro: The Pro-Saude Study. Revista Brasileira de Epidemilogia, 12(3), 1-8.

Roostaee, F., Nikmanesh, Z., Sharifi-Rad, J., Kiani, M., Shahnazi, A. (2016). Relation of religious coping with occupational stress and quality of working life for midwives working in maternity hospitals in Zahedan, Iran. International Journal of Research in Medical Sciences, 4(12), 5329-5333.

Sarafis, P., Rousaki, E., Tsounis, A., Malliarou, M., Lahana, L., Bamidis, P., et al. (2016). The impact of occupational stress on nurses' caring behaviors and their health related quality of life. BMC Nursing, 15, 56.

Shen, S., Yen, M., Yang, S., \& Lee, C. (2016). Insomnia, anxiety, and heart rate variability among nurses working different shift systems in Taiwan. Nursing and Health Sciences, 18, 223-229. 
Wienger, L., Hange, D., Bjorkelund, C., \& Ahlborg, G. (2015). Prevalence of perceived stress and associations to symptoms of exhaustion, depression and anxiety in a working age population seeking primary care - an observational study. BMC Family Practice, 16, 38.
Yazdi, Z., Sadeghniiat-Haghighi, K., Zohal, M. A., \& Elmizadeh, K. (2012). Validity and reliability of the Iranian version of the Insomnia Severity Index. Malaysian Journal of Medical Sciences, 19(4), 31-36. 